\title{
Berufspartnerschaft aus verschiedenen Blickwinkeln
}

Jahreswechsel versprechen immer viel Neues und Spannendes. Auch Der Freie Zahnarzt startet 2016 mit einer Neuerung: Mehrere Fachautoren bereiten in verschiedenen Rubriken ein Schwerpunktthema auf und beleuchten es aus unterschiedlichen Blickwinkeln. Alle zwei Monate wird sich die DFZ-Redaktion unterschiedliche Perspektiven zu einem Thema vornehmen. Zum Start geht es in dieser Ausgabe um zahnärztliche Kooperationen. Obwohl die Einzelpraxis nach wie vor die beliebteste Form der freiberuflichen Selbstständigkeit ist, entscheiden sich vor allem Existenzgründer gerne für eine kooperative Berufsausübung. Doch was gilt es zu beachten, wenn sich ein Zahnarzt mit einem Kollegen zusammentut und eine Berufspartnerschaft eingeht? Gibt es juristische Fallstricke? Wie sieht es mit der Gewinnverteilung aus? Welche besonderen steuerlichen Aspekte kommen auf die Zahnärzte zu? Und wie vermarktet sich das neue Team am besten?

Kompetente Antworten geben die Artikel mit dem blauen Schwerpunktthema-Button auf den Seiten.

\section{Gutachten}

\section{Experten empfehlen Teilkrankschreibungen}

Ein bisschen krank sein und trotzdem arbeiten - das soll nach einem Vorschlag des Sachverständigenrates Gesundheit bald Realität werden. Der konkrete Vorschlag der Experten: Es sollen Teilarbeitsunfähigkeit und Teilkrankengeld eingeführt werden. Laut Gutachten, das im Dezember veröffentlicht wurde, dürfe der Arzt im Krankheitsfall, im Einvernehmen mit dem Patienten, eine teilweise Einsatzfähigkeit bescheinigen. Entsprechend der prozentualen Arbeitsunfähigkeit sollte das Krankengeld in 25-50-75-100-Prozent-Schritten ausgezahlt werden.

Die Gesundheitsweisen begründen die Idee damit, dass das restliche Leistungsvermögen des Arbeitnehmers genutzt werden könne. Die Pläne lösten bei Ärzteschaft, Psychotherapeuten und Öffentlichkeit geteilte Reaktionen aus. Der Vorsitzende des Deutschen Hausärzteverbandes, Ulrich Weigeldt, erklärte gegenüber der „Ärzte Zeitung“, dass die Umsetzung in der Praxis zusätzliche Last für Ärzte sei. Sie müssten mit dem Patienten über den Prozentsatz des Restleistungsvermögens diskutieren. Im Gegensatz dazu hält die Deutsche Psychotherapeutenvereinigung eine Teilkrankschreibung gerade bei psychischen Erkrankungen für sinnvoll. In einer Online-Umfrage unter den Lesern der „Ärzte Zeitung“ sprachen sich zwei Drittel der Teilnehmer gegen Teilarbeitsfähigkeit und Teilkrankengeld aus. Das klare Urteil der Mehrheit der 520 Umfrageteilnehmer: „Wer krank geschrieben wird, ist arbeitsunfähig."

Das Bundesgesundheitsministerium hatte das Gutachten im vergangenen Jahr beim Sachverständigenrat in Auftrag gegeben. Hintergrund: Zwischen 2006 und 2014 haben sich die Kranken-
geld-Ausgaben der gesetzlichen Krankenversicherungen fast verdoppelt. Während die Kassen 2006 noch 5,7 Milliarden Euro Krankengeld zahlten, waren es im vergangenen Jahr bereits 10,6 Milliarden Euro.

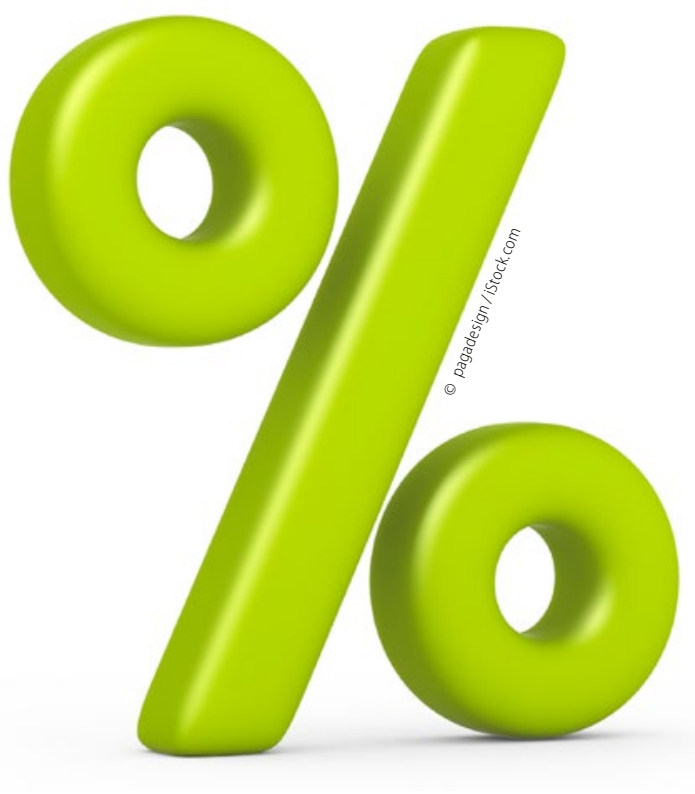

Vorwurf der Untreue

\section{Gesundheitsministerium zeigt Ex-KBV-Chef an}

Das Bundesministerium für Gesundheit (BMG) hat bei der Berliner Staatsanwaltschaft Anzeige gegen den ehemaligen Vorsitzenden der Kassenärztlichen Bundesvereinigung (KBV), Andreas Köhler, erhoben. Nach einem Bericht der Süddeutschen Zeitung lautet der Vorwurf „Untreue in besonders schwerem Fall“. Angeblich zahlte die KBV Köhler zusätzlich zum Gehalt einen Mietkostenzuschuss in Höhe von insgesamt 96.000 Euro. Das Ministerium habe jetzt eingegriffen, damit der Vorfall nicht verjähre. Bereits im Sommer wurde bekannt, dass die KBV ihren einstigen Chef aufforderte, einen Teil seiner Pensionszahlungen zurückzuzahlen. Zuvor hatte das BMG die KBV dazu aufgefordert und eine Frist gesetzt. 